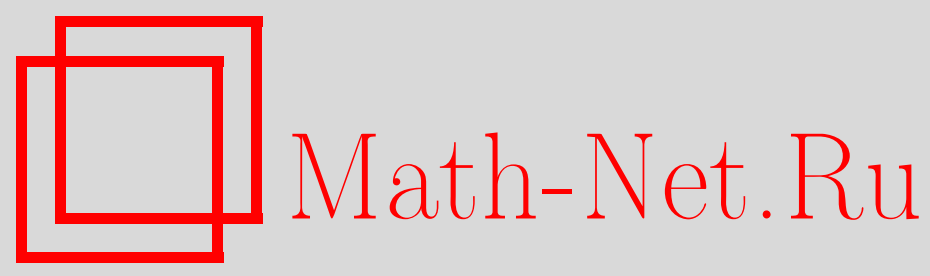

В. Т. Фоменко, О метриках, возникающих на поверхностях постоянной средней кривизны, Матем. заметки, 2005, том 77, выпуск 4, 617-622

DOI: https://doi.org/10.4213/mzm2510

Использование Общероссийского математического портала Math-Net.Ru подразумевает, что вы прочитали и согласны с пользовательским соглашением http://www . mathnet.ru/rus/agreement

Параметры загрузки:

IP : 54.89 .56 .158

26 апреля 2023 г., 16:26:00

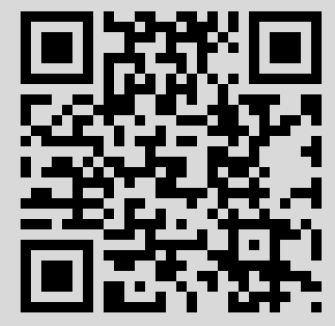




\section{О МЕТРИКАХ, ВОЗНИКАЮЩИХ НА ПОВЕРХНОСТЯХ ПОСТОЯННОЙ СРЕДНЕЙ КРИВИЗНЫ}

\section{В. Т. Фоменко}

В работе сформулированы необходимые и достаточные условия на риманову метрику, гарантируюшие возможность ее погружения в трехмерное пространство постоянной кривизны в виде поверхности постоянной средней кривизны. Эта теорема является обобщением нескольких классических результатов, в частности, теоремы Риччи, которая дает описание метрик, возникающих на минимальных поверхностях в $\mathbb{R}^{3}$.

Библиография: 6 названий.

1. Если опустить замкнутьй проволочньй контур в мыльную воду, а затем аккуратно его извлечь оттуда, то на контуре останется мыльная пленка. Если подуть на пленку, то можно получить мыльный пузырь. В обоих случаях будем говорить, что мы имеем дело с мыльными пленками. Толщина мыльной пленки очень мала, и потому в окрестности каждой регулярной точки мыльную пленку можно рассматривать как ориентируемую двумерную поверхность $S$. Если $P, p$ - давления, действующие на мыльную пленку соответственно с внешней и внутренней стороны пленки, то имеет место формула Лапласа $P-p=2 q H$, где $H$ - средняя кривизна поверхности $S, q-$ сила поверхностного натяжения пленки $S, q=$ const. Для мыльных пленок, находящихся в равновесии, можно считать, что $P-p=$ const, и потому $H=$ const. Это означает, что мыльная пленка всегда представляет собой на регулярных частях поверхность постоянной средней кривизны $H=H_{0}, H_{0}=$ const. Если мыльная пленка натянута на замкнутьй проволочный контур, то можно считать, что $P=p$, и потому $H \equiv 0$, т.е. мыльная пленка, натянутая на замкнутьй контур, представляет собой минимальную поверхность. Для мыльного пузыря, находящегося в равновесии, $P \neq p$, и потому $H \neq 0$. Следовательно, мыльньй пузырь или его часть является поверхностью постоянной средней кривизны $H=H_{0}$, $H_{0}=$ const $\neq 0$.

Естественно, что пространственная форма мыльной пленки существенно зависит от формы контура, на который она натянута, или способа получения мыльного пузыря. В частности, меняя внешнюю форму контура, можно менять внешнюю форму мыльной пленки. При этом, вообще говоря, будет меняться и внутренняя геометрия пленки. Так как внутренняя геометрия поверхности определяется ее первой квадратичной формой $d s^{2}$, естественно поставить вопрос: каким условиям должна удовлетворять двумерная метрика $d s^{2}$, чтобы она являлась первой квадратичной формой мыльной пленки? Другими словами, каким условиям должна удовлетворять двумерная метрика $d s^{2}$, 
чтобы ее можно было погрузить в евклидово пространство $E^{3}$ в виде поверхности постоянной средней кривизны $S\left(H_{0}\right)$, в частности, в виде минимальной поверхности?

Перечислим результаты, полученные ранее в этом направлении.

Пусть $d s^{2}$ - метрика класса $C^{4}$, определенная на плоскости. Говорят, что метрика $d s^{2}$ удовлетворяет условию Риччи, если кривизна $K_{s}$ этой метрики отрищательна: $K_{s}<0$, и если новая метрика $d \sigma^{2}=\sqrt{-K_{s}} d s^{2}$ является плоской, т.е. если кривизна $K_{\sigma}$ метрики $d \sigma^{2}$ тождественно равна нулю: $K_{\sigma} \equiv 0$. Риччи первым обнаружил, что каждая метрика $d s^{2}$, удовлетворяющая этому условию, может быть реализована в $E^{3}$ в виде минимальной поверхности [1, с. 124]. Верно и обратное утверждение: каждая метрика $d s^{2}$ минимальной поверхности отрицательной гауссовой кривизны в $E^{3}$ удовлетворяет условию Риччи. Для поверхностей постоянной средней кривизны $H=H_{0}$, $H_{0}=$ const, имеет место аналогичное утверждение, отмеченное без доказательства Ю.А. Аминовым [2, с. 33]: если $d s^{2}$ - метрика поверхности постоянной средней кривизны $H_{0}$ в $E^{3}$, то метрика $d \sigma^{2}=\sqrt{H_{0}^{2}-K_{s}} d s^{2}$ является плоской. М. Пинл показал, что в $E^{4}$ существуют минимальные поверхности, на которых условие Риччи не выполняется [3]. В тоже время Н. В. Лоусон [4] (см. также [5]) доказал следуюший результат: если кривизна $K_{s}$ метрики $d s^{2}$ класса $C^{4}$, определенной на плоскости, удовлетворяет условию $K_{s}<1 / r^{2}$ для некоторой константы $r>0$, и если метрика $d \sigma^{2}=\sqrt{1 / r^{2}-K_{s}} d s^{2}$ является плоской, то метрика $d s^{2}$ может быть реализована в виде непрерьвного однопараметрического семейства минимальных поверхностей в евклидовой сфере $S^{3}(r)$ кривизны $1 / r^{2}$.

Аналогом условия Риччи может служить следующее условие, которое мы назьваем условием Нордена. Пусть $d s^{2}$ - метрика класса $C^{4}$, определенная на плоскости. Будем говорить, что метрика $d s^{2}$ удовлетворяет условию Нордена, если кривизна метрики отрицательна: $K_{s}<0$, и если кривизна $K_{\tau}$ новой метрики $d \tau^{2}=-K_{s} d s^{2}$ тождественно равна единице: $K_{\tau} \equiv+1$. А. П. Норден доказал [6, с. 237], что в евклидовом пространстве $E^{3}$ всякая поверхность класса $C^{4}$ с метрикой, удовлетворяющей условию Нордена, локально наложима на минимальную поверхность.

В настоящей работе приводится условие, обобщающее условия Риччи, Нордена и Лоусона, необходимые и достаточные для того, чтобы двумерная метрика $d s^{2}$ класса $C^{4}$, заданная в односвязной плоской области, допускала изометрическоепогружение в трехмерное риманово пространство $R^{3}\left(c_{0}\right)$ постоянной кривизны $c_{0}, c_{0}=$ const, в виде поверхности постоянной средней кривизны $H=H_{0}, H_{0}=$ const.

2. Пусть $R^{3}\left(c_{0}\right)$ - трехмерное риманово пространство постоянной кривизны $c_{0}, c_{0}=$ const, $H_{0}, \alpha, \alpha \neq 0,-$ заданные действительные числа. Имеет место следующая теоремa.

Теорема. Двумерная метрика $d s^{2}$ класса $C^{4}$, заданная в односвязной плоской области, допускает изометрическое погружение в пространство $R^{3}\left(c_{0}\right)$ в виде поверхности постоянной средней кривизны $H_{0}$, не содержащей омбилических точек, тогда и только тогда, когда кривизна $K_{s}$ удовлетворяет условию $K_{s}<H_{0}^{2}+c_{0}$, а кривизна $K_{\tau_{\alpha}}$ новой метрики $d \tau_{\alpha}^{2}=\left(-K_{s}+H_{0}^{2}+c_{0}\right)^{\alpha} d s^{2}$ вычисляется по формуле

$$
K_{\tau_{\alpha}}=\frac{(1-2 \alpha) K_{s}}{\left(-K_{s}+H_{0}^{2}+c_{0}\right)^{\alpha}} .
$$


При әтом изометрическое погружение метрики $\mathrm{ds}^{2}$ в $R^{3}\left(c_{0}\right)$ осуществляется $c$ точностью до движения в виде непрерывно зависящего от параметра $t, t \in[0,2 \pi)$, однопараметрического семейства $\left\{S_{t}\left(H_{0}\right)\right\}$ поверхностей $S_{t}\left(H_{0}\right)$ постоянной средней кривизны $H_{0}$, попарно не конгруентных друг другу при различных значениях параметра $t$.

Отметим, что в случае $\alpha=1 / 2, H_{0}=0, c_{0}=0$ утверждение теоремы совпадает с результатом Риччи, в случае $\alpha=1, H_{0}=0, c_{0}=0-$ с результатом Нордена, в случае $\alpha=1 / 2, H_{0}=0, c_{0}=1 / r^{2}-$ с результатом Лоусона, а при $\alpha=1 / 2, H_{0} \neq 0, c_{0}=0$ получаем утверждение, отмеченное Аминовым.

Ниже мы формулируем частные случаи этой теоремы.

ОБОБЩЕнНАЯ теорема РИччИ. Пусть $H_{0}, c_{0}-$ заданные числа. Для того, чтобь метрика $d s^{2}$ класса $C^{4}$ кривизны $K_{s}, K_{s}<H_{0}^{2}+c_{0}$, заданная в односвязной плоской области, допускала изометрическое погружение в пространство $R^{3}\left(c_{0}\right)$ в виде поверхности постоянной средней кривизны $H_{0}$ без омбилических точек, необходимо и достаточно, чтобы метрика $d \sigma^{2}=\sqrt{-K_{s}+H_{0}^{2}+c_{0}} d s^{2}$ была плоской, т.е. чтобы кривизна $K_{\sigma}$ метрики $d \sigma^{2}$ тожсдественно равнялась нулю: $K_{\sigma} \equiv 0$.

ОБОБЩЕННАЯ ТЕОРЕМА НОРДЕНА. Для того, чтобы метрика $d^{2}$ класса $C^{4}$ отрицательной кривизны $K_{s}<0$, заданная в односвязной плоской области, допускала изометрическое погружение в пространство $R^{3}\left(c_{0}\right)$ неположительной кривизны $c_{0} \leqslant 0$ в виде поверхности постоянной средней кривизны $H=\sqrt{-c_{0}}$ без омбилических точек, необходимо и достаточно, чтобы кривизна $K_{\tau}$ метрики $d \tau^{2}=\left(-K_{s}\right) d s^{2}$ била тождественно равна единице: $K_{\tau} \equiv+1$.

3. ДоКАЗАТЕЛЬСТво ТЕОРЕМЫ. Необходимость. Пусть поверхность $S\left(H_{0}\right)$ постоянной средней кривизны $H=H_{0}$ отнесена к линиям кривизны $(x, y)$. Так как поверхность $S\left(H_{0}\right)$ не содержит омбилических точек, то кривизна $K_{s}$ метрики поверхности удовлетворяет условию $K_{s}<H_{0}^{2}+c_{0}$. Основные квадратичные формы поверхности $S\left(H_{0}\right)$ в линиях кривизны имеют вид

$$
d s^{2}=g_{11} d x^{2}+g_{22} d y^{2}, \quad I I=b_{11} d x^{2}+b_{22} d y^{2} .
$$

Обозначим через $k_{1}, k_{2}$ - главные кривизны поверхности $S\left(H_{0}\right)$. Тогда имеют место формулы

$$
k_{1}=\frac{b_{11}}{g_{11}}, \quad k_{2}=\frac{b_{22}}{g_{22}}, \quad H_{0}=\frac{k_{1}+k_{2}}{2}, \quad K=k_{1} k_{2},
$$

где $K$ - гауссова кривизна поверхности $S\left(H_{0}\right), K=K_{s}-c_{0}$. Обозначим через $G_{i j}$ коэффициенты квадратичной формы $d \tau_{\alpha}^{2}=\left(-K_{s}+H_{0}^{2}+c_{0}\right)^{\alpha} d s^{2}$. Тогда имеем

$$
G_{11}=\left(H_{0}-k_{1}\right)^{2 \alpha} g_{11}, \quad G_{12}=0, \quad G_{22}=\left(H_{0}-k_{2}\right)^{2 \alpha} g_{22}
$$

Подсчитаем кривизну $K_{\tau_{\alpha}}$ метрической формы $d \tau_{\alpha}^{2}$, используя формулу

$$
K_{\tau_{\alpha}}=-\frac{1}{2 \sqrt{G_{11} G_{22}}}\left\{\frac{\partial}{\partial y}\left(\frac{1}{\sqrt{G_{11} G_{22}}} \frac{\partial}{\partial y} G_{11}\right)+\frac{\partial}{\partial x}\left(\frac{1}{\sqrt{G_{11} G_{22}}} \frac{\partial}{\partial x} G_{22}\right)\right\} \text {. }
$$


Воспользуемся уравнениями Кодацщи, записанными в виде

$$
\frac{\partial k_{1}}{\partial y}=\frac{1}{2 g_{11}} \frac{\partial g_{11}}{\partial y}\left(k_{2}-k_{1}\right), \quad \frac{\partial k_{2}}{\partial x}=\frac{1}{2 g_{22}} \frac{\partial g_{22}}{\partial x}\left(k_{1}-k_{2}\right) .
$$

Имеем

$$
\begin{aligned}
K_{\tau_{\alpha}}= & -\frac{1}{2 \sqrt{G_{11} G_{22}}}\left\{\frac{\partial}{\partial y} \frac{2 \alpha\left(H_{0}-k_{1}\right)^{2 \alpha-1} g_{11}\left(-\frac{\partial k_{1}}{\partial y}\right)+\frac{\partial g_{11}}{\partial y}\left(H_{0}-k_{1}\right)^{2 \alpha}}{\sqrt{g_{11} g_{22}}\left(H_{0}-k_{2}\right)^{2 \alpha}}\right. \\
& \left.+\frac{\partial}{\partial x} \frac{2 \alpha\left(H_{0}-k_{2}\right)^{2 \alpha-1} g_{22}\left(-\frac{\partial k_{2}}{\partial x}\right)+\frac{\partial g_{22}}{\partial x}\left(H_{0}-k_{2}\right)^{2 \alpha}}{\sqrt{g_{11} g_{22}}\left(H_{0}-k_{1}\right)^{2 \alpha}}\right\} .
\end{aligned}
$$

Так как $H_{0}^{2}-K=\left(\left(k_{1}-k_{2}\right) / 2\right)^{2}, K=K_{s}-c_{0}$, последнее соотношение принимает вид

$$
\begin{aligned}
K_{\tau_{\alpha}} & =-\frac{(1-2 \alpha)}{\left(-K_{s}+H_{0}^{2}+c_{0}\right)^{\alpha} \sqrt{g_{11} g_{22}}}\left\{\frac{\partial}{\partial y}\left(\frac{1}{\sqrt{g_{11} g_{22}}} \frac{\partial g_{11}}{\partial y}+\frac{\partial}{\partial x} \frac{1}{\sqrt{g_{11} g_{22}}} \frac{\partial g_{22}}{\partial x}\right)\right\} \\
& =\frac{(1-2 \alpha) K_{s}}{\left(-K_{s}+H_{0}^{2}+c_{0}\right)^{\alpha}}
\end{aligned}
$$

что и требовалось доказать.

Достаточность. Зададим метрику $d s^{2}$ в изотермических координатах $(x, y)$. Тогда $d s^{2}=E(x, y)\left(d x^{2}+d y^{2}\right),(x, y) \in D$, где $D$ - некоторая односвязная область параметрической плоскости. Для того, чтобы доказать возможность погружения метрики $d s^{2}$ в пространство $R^{3}\left(c_{0}\right)$, докажем разрешимость системы уравнений Гаусса-Кодацци, которая в изотермических координатах имеет вид

$$
\begin{gathered}
b_{11} b_{22}-b_{12}^{2}=\left(K_{s}-c_{0}\right) E^{2}, \quad K_{s}=-\frac{1}{2 E} \Delta \ln E, \quad \Delta=\frac{\partial^{2}}{\partial x^{2}}+\frac{\partial^{2}}{\partial y^{2}} \\
\left(b_{11}-E H_{0}\right)_{y}-\left(b_{12}\right)_{x}=0, \quad\left(b_{22}-E H_{0}\right)_{x}-\left(b_{12}\right)_{y}=0 .
\end{gathered}
$$

Здесь $b_{11}, b_{12}, b_{22}$ - искомые функции, которые в дальнейшем будут служить коэффициентами второй квадратичной формы погружения метрики $d s^{2}$ в $R^{3}\left(c_{0}\right)$ в виде поверхности постоянной средней кривизны $H_{0}$. В связи с этим коэффициенты $b_{i j}$ связаны также соотношением

$$
b_{11}+b_{22}=2 H_{0} E \text {. }
$$

Полагая $b_{11}=\Lambda_{0}+H_{0} E, b_{12}=M_{0}, b_{22}=-\Lambda_{0}+H_{0} E$, из уравнений $(2),(3)$ находим, что функция $\Phi(z)=M_{0}+i \Lambda_{0}, z=x+i y, i^{2}=-1$, является аналитической функцией комплексного переменного $z$ в области $D$. В силу уравнения Гаусса $(1)$ функция $\Phi(z)$ удовлетворяет условию

$$
|\Phi(z)|^{2}=M_{0}^{2}+\Lambda_{0}^{2}=E^{2}\left(-K_{s}+H_{0}^{2}+c_{0}\right) .
$$

Так как правая часть этого соотношения есть известная функция, она может служить квадратом модуля аналитической функции. Другими словами, нужно убедиться 
в том, что $\ln \left(E^{2}\left(-K_{s}+H_{0}^{2}+c_{0}\right)\right)$ есть гармоническая функция при сделанных в теореме предположениях. Посчитаем $\Delta \ln \left(E^{2}\left(-K_{s}+H_{0}^{2}+c_{0}\right)\right)$. Имеем в силу формулы $K_{s}=-\frac{1}{2 E} \Delta \ln E$ соотношение

$$
\begin{aligned}
\Delta \ln \left(E^{2}\left(-K_{s}+H_{0}^{2}+c_{0}\right)\right) & =2 \Delta \ln E+\Delta \ln \left(-K_{s}+H_{0}^{2}+c_{0}\right) \\
& =-4 E K_{s}+\Delta \ln \left(-K_{s}+H_{0}^{2}+c_{0}\right) .
\end{aligned}
$$

Подсчитаем кривизну $K_{\tau_{\alpha}}$ метрики $d \tau_{\alpha}^{2}=\left(-K_{s}+H_{0}^{2}+c_{0}\right)^{\alpha} d s^{2}$ в изотермических координатах. Имеем

$$
\begin{aligned}
K_{\tau_{\alpha}} & =-\frac{1}{2\left(-K_{s}+H_{0}^{2}+c_{0}\right)^{\alpha} E} \Delta \ln \left(\left(-K_{s}+H_{0}^{2}+c_{0}\right)^{\alpha} E\right) \\
& =-\frac{\alpha \Delta \ln \left(-K_{s}+H_{0}^{2}+c_{0}\right)+\Delta \ln E}{2\left(-K_{s}+H_{0}^{2}+c_{0}\right)^{\alpha} E}
\end{aligned}
$$

Так как по условию теоремы

$$
K_{\tau_{\alpha}}=\frac{K_{s}(1-2 \alpha)}{\left(-K_{s}+H_{0}^{2}+c_{0}\right)^{\alpha}}
$$

отсюда находим $\alpha\left(\Delta \ln \left(-K_{s}+H_{0}^{2}+c_{0}\right)-4 E K_{s}\right)=0$. Так как $\alpha \neq 0$, то $\Delta \ln \left(-K_{s}+\right.$ $\left.H_{0}^{2}+c_{0}\right)=4 E K_{s}$ и потому функция $\ln E^{2}\left(-K_{s}+H_{0}^{2}+c_{0}\right)$ является гармонической. Это означает, что уравнение Гаусса (4) будет удовлетворено, если функцию $|\Phi(z)|$ выбрать по формуле $|\Phi(z)|=E \sqrt{-K_{s}+H_{0}^{2}+c_{0}}, z \in D$. Зная $|\Phi(z)|$, найдем $\Phi(z)=|\Phi(z)| \times$ $\exp (i \arg \Phi(z))$, где

$$
\arg \Phi(z)=\int_{\left(x_{0}, y_{0}\right)}^{(x, y)}\left[-(\ln |\Phi(z)|)_{y} d x+(\ln |\Phi(z)|)_{x} d y\right]+t,
$$

$\left(x_{0}, y_{0}\right)$ - некоторая фиксированная точка области $D, z=x+i y, t$-вещественньй параметр, $t \in[0,2 \pi) ;$ интегрирование ведется по любому пути, соединяющему точку $\left(x_{0}, y_{0}\right)$ с точкой $(x, y)$. В силу односвязности области $D$ криволинейный интеграл не зависит от пути интегрирования и потому существует. Зная функцию $\Phi(z)$, однозначно находим коэффищиенты $b_{i j}$ по формулам

$$
b_{11}=\operatorname{Im} \Phi(z)+H_{0} E, \quad b_{12}=\operatorname{Re} \Phi(z), \quad b_{22}=-\operatorname{Im} \Phi(z)+H_{0} E, \quad z \in D .
$$

Коэффициенты $b_{i j}$, определенные формулами (5), непрерывно зависят от параметра $t$, причем для различных значений $t$ из промежутка $[0,2 \pi)$ значения $b_{i j}$ различны. Кроме того, для любых значений $t, t \in[0,2 \pi)$, коэффициенты $b_{i j}$, определенные формулами (5), удовлетворяют условию (3). Это означает, что для любого фиксированного значения параметра $t, t \in[0,2 \pi)$, квадратичные формы

$$
d s^{2}=E\left(d x^{2}+d y^{2}\right), \quad I I=b_{11} d x^{2}+2 b_{12} d x d y+b_{22} d y^{2}, \quad(x, y) \in D,
$$

определяют в пространстве $R^{3}\left(c_{0}\right)$ с точностью до движения единственную поверхность $S_{t}\left(H_{0}\right)$ постоянной средней кривизны $H=H_{0}=$ const. Полученное семейство $\left\{S_{t}\left(H_{0}\right)\right\}$, $t \in[0,2 \pi)$, поверхностей $S_{t}\left(H_{0}\right)$ непрерьвно зависит от параметра $t$ и исчерпьвает все возможные реализации метрики $d s^{2}$ в $R^{3}\left(c_{0}\right)$ в виде поверхностей постоянной средней кривизны $H=H_{0}=$ const. Теорема доказана. 
ПримеР. Метрика минимальной без точек уплощения поверхности отрицательной кривизны в $E^{3}$, заданная в односвязной области, допускает изометрическоепогружение в пространство $R^{3}\left(c_{0}\right), c_{0}<0$, в виде однопараметрического семейства, непрерьвно зависяшего от параметра, поверхностей постоянной средней кривизны $H=\sqrt{\left|c_{0}\right|}$.

\section{СПИСОК ЦИТИРОВАННОЙ ЛИТЕРАТУРЫ}

[1] Blaschke W. Einführung in die Differentialgeometrie. Berlin: Springer, 1950.

[2] Аминов Ю. А. Минимальные поверхности. Харьков: Изд-во ХГУ, 1978.

[3] Pinl M. Über einen Satz von G. Ricci-Curbastro und die Gaussche Krümmung der Minimalflächen // Arch. Math. 1953. V. 4. P. 369-373.

[4] Lawson H. B. Jr. Some intrinsic characterizations of minimal surfaces // J. Anal. Math. 1971. V. 24. P. 151-161.

[5] Lawson H. B. Jr. Minimal Varieties in Constant Curvature Manifolds. Ph. D. Thesis. Stanford: Stanford University, 1968.

[6] Норден А. П. Теория поверхностей. М.: ГИТТЛ, 1956.

Таганрогский государственный педагогический институт 